\title{
LEFT ALMOST PERIODICITY DOES NOT IMPLY RIGHT ALMOST PERIODICITY
}

\author{
BY T. S. $\mathrm{WU}^{1,2}$
}

Communicated by G. A. Hedlund, August 16, 1965

Let $G$ be a topological group. A real valued continuous function $f$, defined on $G$, is left \{right $\}$ almost periodic iff for any $\epsilon>0$, there is a left $\{$ right $\}$ syndetic subset ${ }^{3} A$ of $G$ such that $|f(a x)-f(x)|<\epsilon$ for all $a \in A, x \in G$. In this note, we shall show that a left almost periodic function is not necessarily right almost periodic even if the group $G$ is a Lie group. This answers a problem in [3]. For the notions of almost periodic functions, we refer to $[1],[4] . C(X)$ denotes the set of all continuous real valued functions on the topological space $X$.

Definition 1. Let $N$ be a closed subgroup of a topological group $G$. We say that $N$ splits in $G$ if $N$ is normal in $G$ and there is a closed subgroup $C$ such that

(i) $N \cap C=\{e\}, e$ the identity of $G$.

(ii) $G=C N$.

(iii) The mapping $(c, n) \rightarrow c n$ is a homeomorphism of $C X N$ onto $G$. In this case $G$ is said to be the semidirect product of $N$ and $C$ [5], [6].

Proposition 1. Assume $G$ is a semidirect product of a compact normal subgroup $N$ and a subgroup $C$. Let $f \in C(N)$. Define $F$ on $G$ by $F(c n)=f(n)$. Then $F \in C(G)$, and $F$ is left almost periodic.

Proof. It is clear that $F$ is well defined and belongs to $C(G)$. Let $\epsilon>0,|F(c n)-F(n)|=|f(n)-f(n)|=0<\epsilon$. Since $C N=G, C$ is left syndetic. Thus $F$ is left almost periodic. In fact, $F$ is left periodic in the sense of [4].

Proposition 2. In addition to the assumption and notations of Proposition 1 , we assume that there are elements $n \in N, n \neq e$, and a net $\left\{g_{\nu}\right\}$ in $G$ such that $g_{\nu} n g_{\nu}^{-1} \rightarrow e$. If $f \in C(N)$, with $f(n) \neq f(e)$, then $F$ is not right almost periodic.

Proof.

$$
\left|F\left(g_{\nu} n g^{-1} g_{p}\right)-F\left(g_{\nu}\right)\right|=\left|F\left(g_{\nu} n\right)-F\left(g_{\nu} e\right)\right|=|f(n)-f(e)|=a \neq 0,
$$

${ }^{1}$ I am grateful to Professor R. W. Bagley for his encouragement during my preparation of this work.

2 This work was supported partially under the contract NGR 10-007-005.

3 A subset $S$ of $G$ is left \{right $\}$ syndetic [4] iff there exists a compact subset $K$ of $G$ so that $G=S K\{G=K S\}$. 
it follows that $F$ is not right uniformly continuous. Because $F$ is left almost periodic, by Theorem 4.61 in [4], $F$ is not right almost periodic.

DEFINITION 2. Let $\phi$ be an automorphism of a compact group $N$. We say that $\phi$ is expansive iff there is a neighborhood $V$ of the identity $e$ in $N$ such that for any $x, y \in N$; there is an integer $i$, with $\phi^{i}\left(x y^{-1}\right) \notin V$. For the notion of expansive homeomorphisms, we refer to [4]. For the existence of expansive automorphisms on Lie groups and manifolds, see [2], [7], [8]. It is well known that there exist expansive automorphisms on the torus group.

Proposition 3. Let $\phi$ be an expansive automorphism on an infinite compact group $N$. Then there is $n \in N$, and a sequence of integers $\alpha_{\nu}$ such that $\phi^{\alpha_{\nu}}(n)$ converges to $e$.

Proof. Since $\phi(e)=e, e$ is a fixed point under $\phi$. By 10.37 in [4], the result follows.

Construction. Let $N$ be an infinite compact group, and $\phi$ an expansive automorphism on $N$. We use $C$ to denote the discrete free group generated by $z$, a symbol not in $N$, and let $\mu$ be the identity in $C$. Let $G$ be the Cartesian product of $C$ and $N$ with the product topology. Define the group operation in $G$ by

$$
\left(z^{i}, n\right)\left(z^{j}, m\right)=\left(z^{i+j}, \phi^{j}(n) m\right) .
$$

It is not hard to see that $G$ is a topological group under such multiplication, $[5$, p. 6, p. 58], and $G$ is the semidirect product of the compact normal subgroup $N^{\prime}=\{(\mu, n) \mid n \in N\}$ and the subgroup $C^{\prime}$ $=\{(x, e) \mid x \in C\}$. Moreover,

$$
\begin{aligned}
(z, e)^{-1}(\mu, n)(z, e) & =\left(z^{-1}, e\right)(\mu, n)(z, e)=\left(z^{-1}, n\right)(z, e)=(\mu, \phi(n) e) \\
& =(\mu, \phi(n)) .
\end{aligned}
$$

Thus, the inner automorphism induced by $(z, e)$ on $N^{\prime}$ is an expansive automorphism. By the above propositions, there exists a left almost periodic function which is not right almost periodic.

REMARKS. It is not hard to prove that every left almost periodic function on $G$ constructed above is left periodic in the sense of [4]. Also, the set of all left almost periodic functions on $G$ does not separate points.

Added in proof. Recently, we have shown there exist connected Lie groups whose left almost periodic functions which is not right almost periodic separates points. The detail will appear elsewhere. 


\section{REFERENCES}

1. R. Ellis, Equicontinuity and almost periodic functions, Proc. Amer. Math. Soc., 10 (1959), 637-643.

2. M. Eisenberg, Dissertation, Wesleyan University, Middletown, Conn., 1965.

3. W. H. Gottschalk, Minimal sets: an introduction to topological dynamics, Bull. Amer. Math. Soc. 64 (1958), 336-351.

4. W. H. Gottschalk and G. A. Hedlund, Topological dynamics, Amer. Math. Soc. Colloq. Publ. Vol. 36, Amer. Math. Soc., Providence, R. I., 1955.

5. E. Hewitt and K. A. Ross, Abstract harmonic analysis. I, Academic Press, New York, 1963.

6. K. H. Hofmann and P. Mostert, Splitting in topological groups, Mem. Amer. Math. Soc. No. 43 (1963).

7. W. Reddy, The existence of expansive homeomorphisms on manifolds, Dissertation, Syracuse University, Syracuse, N. Y., 1964.

8. T. S. Wu, Expansive automorphisms in compact groups, Math. Scand. (to appear).

\section{WESLEYAN UNIVERSITY}

